\title{
Lateral transport in quasiperiodically ordered layered media with isotropic randomness
}

\author{
Man-Chung Chan and Zhao-Qing Zhang \\ Department of Physics, Hong Kong University of Science and Technology, Clear Water Bay, \\ Kowloon, Hong Kong, People's Republic of China
}

Received 26 June 2001, in final form 13 November 2001

Published 18 December 2001

Online at stacks.iop.org/WRM/12/201

\begin{abstract}
We investigate the lateral wave transport in quasiperiodically ordered layer media with isotropic randomness. As an example, we consider the case of the Fibonacci sequence and study the ergodic properties in such systems. From the results of the channel occupation number of nine generations, we find that the wave transport in such systems falls between the transport of anisotropic hopping systems and that of randomly layered media and can be associated with a fractal dimension that can be tuned according to the strength of the layer coupling. The origin of this fractal dimensionality is attributed to the interplay between the quasiperiodic ordering in the layer direction and the presence of isotropic randomness in the system.
\end{abstract}

\section{Introduction}

Randomly layered media with isotropic randomness represent an interesting class of systems for wave transport [1-4]. Physical examples of such systems are the Earth's subsurface and random superlattices with lateral inhomogeneities. In the absence of isotropic randomness, a randomly layered medium can be considered as a one-dimensional (1D) system for the wave propagation in the layering direction. Since all states are localized in one-dimensional random media, the transmission coefficient decays exponentially with increasing sample thickness. Since the system is a translational invariant in the transverse plane, each localized state in the layering direction provides a free channel (waveguide) for the wave propagation in the transverse plane. Although these channels are independent, nevertheless, they can overlap among themselves due to the overlaps of 1D localized states. Due to the overlap of channels, the transport behaviour in such systems changes completely and becomes entirely nontrivial when isotropic randomness is present. The presence of isotropic randomness is inevitable in any real system. In the case of random superlattices, it can arise from interdiffusion as well as lateral roughness at the interface. It is possible to use $\mathrm{x}$-ray scattering to characterize various kinds of disorder in disordered superlattices [5]. 
Unlike layer randomness, an isotropic impurity scatters a wave into all different directions. In the layering direction, the presence of isotropic randomness scatters the wave away from the incident direction. This reduces the interference and localization effects in the layering direction and, therefore, enhances the transmission. On the other hand, in the lateral direction, an isotropic impurity can give rise to two independent scattering mechanisms. Let us consider a wave propagating in a particular channel. The presence of isotropic randomness can scatter the wave away from the propagating channel into any other channels that have overlaps with the propagating channel. It can also scatter the wave within the propagating channel. Thus, both inter- and intra-channel scatterings can occur due to the isotropic randomness. The former causes channel hoppings, which facilitate the wave transport in the lateral direction, while the latter tends to localize the wave propagation in each channel. Thus, the competition between these two scattering strengths determines the nature of the transport in such systems.

The relative magnitudes of these two scattering processes are dictated by the degree of overlap among different channel wavefunctions. If the overlap of wavefunctions is large, channel hoppings are strong and a wave can hop among different channels as it propagates in the lateral direction. However, if the overlap is small, wave propagation is mainly confined to a particular channel. Furthermore, since all waves are localized in a 1D or 2D random medium, wave propagation can also become localized in that channel. Thus, the properties of channel wavefunctions are crucial in the determination of the lateral transport in a randomly layered medium when isotropic randomness is present. The degree of channel overlap depends on the layer randomness as well as the strength of the layer coupling. When layer randomness is large or layer coupling is weak, the channel wavefunctions are more localized in the layering direction. In this case the channel overlaps are small and the intra-channel scattering is dominant. However, when the layer randomness is small or the layer coupling is strong, channels are more extended. Due to large channel overlaps, channel hoppings become dominant, and this facilitates the lateral transport.

Although the wave transport in randomly layered medium with isotropic randomness is different in the layering and lateral directions, these systems are entirely different in nature from other types of system that also possess anisotropic transport behaviour, i.e. anisotropic hopping systems [6]. In anisotropic hopping systems, only isotropic randomness appears and the anisotropy arises purely from the difference in the hopping integrals in different directions. Due to the absence of layer randomness all channels fully overlap. In fact, it has been shown that the anisotropic hopping system behaves like an ordinary isotropic system when the transport in each direction is properly rescaled [6].

Wave transport in randomly layered media with isotropic randomness has been studied in the past. Very rich transport behaviour not seen in other disordered systems has been revealed [1-4]. The study of 3D randomly layered media with isotropic randomness in the past has shown that, when the ratio of the strength of layer randomness to that of isotropic randomness is larger than a certain critical value, the system is $1 \mathrm{D}$-like and all states are localized [1]. When the ratio is smaller than the critical value, the transport is 3D-like and the Anderson localization transition can occur. In the later studies on the lateral transport in 2D systems, it has been found that there exists an equilibration length above which the channel distribution reaches a steady state and is independent of the incident channel. Furthermore, the transport in the asymptotic region is nonergodic in the channel space due to the existence of a dominant channel with the largest channel speed [2,3]. A recent finite-size scaling study in 2D has revealed an interesting dimensional-crossover behaviour. At small length scales, the transport in randomly layered media with isotropic randomness is ergodic and behaves like anisotropic hopping systems. However, as the number of layers in randomly layered media with isotropic randomness is increased, the system crosses over to the nonergodic regime 
and behaves like a 1D system [4]. It is also found that the crossover length increases with the coupling strength between adjacent layers. Thus, all these known results in 2D and 3D have shown explicitly that the role played by the degree of channel overlap is crucial in the determination of wave transport in randomly layered media with isotropic randomness.

Anisotropic hopping systems and randomly layered media with isotropic randomness are the only two classes of anisotropic systems that have been studied so far. A natural question to ask is whether there exist other classes of anisotropic media whose transport falls between that of anisotropic hopping systems and randomly layered media with isotropic randomness? Since the only difference between anisotropic hopping systems and randomly layered media with isotropic randomness is the layer ordering, i.e. the former is ordered while the latter is random, it is expected that a quasiperiodic ordering in the layer direction would produce a lateral transport that is intermediate between anisotropic hopping systems and randomly layered media with isotropic randomness. In this work, we study explicitly the lateral transport in quasiperiodically ordered layered media with isotropic randomness. In the absence of isotropic randomness, a quasiperiodic system is also 1D. The transport properties in the layering direction have been well studied in the past [7-9]. For the case of electronic systems, the effects due to electric field have also been studied [10]. However, the transport properties of such systems have not been investigated when isotropic randomness is present in the systems. In this paper, we consider the simplest case of the Fibonacci sequence. The general features obtained here should also be valid in systems with other types of quasiperiodic ordering. In the absence of isotropic randomness, the channel spectrum for the Fibonacci sequence is known to be a Cantor set and the corresponding wavefunctions are critical and characterized by strong spatial fluctuations $[7,8]$. The transmission study in the layering direction also reveals the self-similar property of a Cantor set [7]. Since the critical channel wavefunctions in such a system fall between extended states in a periodic system and localized states in a randomly layered medium, some novel transport behaviour is expected to arise when isotropic randomness is present. The results of our simulation indicate that the lateral transport in such systems carries a fractal dimension and the dimensionality can be tuned continuously by varying the strength of layer coupling. The origin of this fractal dimensionality is attributed to the interplay between the quasiperiodic ordering in the layer direction and the presence of isotropic randomness in the system.

In section 2, the Hamiltonian of the system is defined. An important matrix has been constructed to describe the inter- and intra-channel scattering processes. It is the structure of this matrix that leads to the fractal-type transport of the system. In section 3, we give a brief description of the numerical method and present the results of our calculations. Discussions and conclusions are given in sections 4 and 5, respectively.

\section{Model Hamiltonian}

To study the lateral transport, we use the following tight-binding Hamiltonian:

$$
H=\sum_{\alpha} \varepsilon_{\alpha}|\alpha\rangle\left\langle\alpha\left|+\sum_{[\alpha, \beta]} t_{\alpha \beta}\right| \alpha\right\rangle\langle\beta|,
$$

where $\alpha$ and $\beta$ are site indices and $[\alpha, \beta]$ indicates two nearest-neighbour sites. The lattice constant is set to unity. The site energy $\varepsilon_{\alpha}$ consists of two parts:

$$
\varepsilon_{\alpha}=\eta_{z}+\delta_{\alpha} .
$$

$\eta_{z}$ describes the layer energy and is a constant for all sites lying in a given layer at fixed $z$. Here $\eta_{z}$ has two values, $\varepsilon_{A}$ and $\varepsilon_{B}$, arranged according to the Fibonacci sequence. $\delta_{\alpha}$ is a 
random number that varies independently from site to site with a flat distribution of width $W$. $W$ measures the strength of the isotropic randomness. In the case of randomly layered media with isotropic randomness, $\eta_{z}$ is also a random number and we can assign another parameter, $W_{1}$, to denote the strength of the layer randomness. In equation (1) we assume that the nearestneighbour hopping integrals are different in different directions. For a 2D system, we choose the $z$-axis as the layering direction and the $x$-axis as the lateral direction. For convenience, we set $t_{x}=1$ as the energy scale. For a given set of layer energies $\left\{\eta_{z}\right\}$, the hopping integral along the $z$-axis $t_{z}$ determines the strength of layer coupling and the degree of channel overlap. Thus, we consider $t_{z}$ as a variable in our calculations. The properties of eigen channels can be obtained by solving the $1 \mathrm{D}$ eigenvalue problem along the $z$ direction. For a system of $N$ layers, we have a set of $N$ normalized channel functions, $\varphi_{n}(z)$, and channel energies $E_{n}$. These wavefunctions act as overlapped channels for the propagation of waves along the $x$ direction. The wave energy can be written as $E=E_{n}+2 \cos k_{n}$, where $k_{n}$ is the wavevector of the $n$th channel for the propagation along the $x$ direction. When $\left|E-E_{n}\right|>2, k_{n}$ is imaginary and the channel is denoted 'evanescent'. When $\left|E-E_{n}\right|<2$ and $k_{n}$ is real, the channel is denoted 'propagating'.

In the presence of isotropic randomness, both inter- and intra-channel scatterings occur. These scattering processes become more transparent if we rewrite the Hamiltonian of equations (1) and (2) in the eigen channel representation [3], i.e. $H=H_{0}+H_{1}$ with

$$
H_{0}=\sum_{n, i} E_{n}\left|n, x_{i}\right\rangle\left\langle n, x_{i}\left|+\sum_{n,[i, j]}\right| n, x_{i}\right\rangle\left\langle n, x_{j}\right|
$$

and

$$
H_{1}=\sum_{n, i} S_{n, n}\left(x_{i}\right)\left|n, x_{i}\right\rangle\left\langle n, x_{i}\left|+\sum_{n \neq m, i} S_{n, m}\left(x_{i}\right)\right| n, x_{i}\right\rangle\left\langle m, x_{i}\right|
$$

where

$$
S_{n, m}\left(x_{i}\right)=\sum_{z} \delta_{z, x_{i}} \varphi_{n}^{*}(z) \varphi_{m}(z) .
$$

Here, $i$ is the site index along the $x$-axis and $[i, j]$ denotes the nearest-neighbour indices $i$ and $j$. The summation in $z$ goes over all $N$ layers. In the absence of isotropic randomness, i.e. $H_{1}=0$, the Hamiltonian $H_{0}$ describes the free wave propagation of each channel $n$ in the lateral direction. In the presence of isotropic randomness, $\delta_{z, x_{i}}$, as defined in equation (2), the function $S_{n, n}\left(x_{i}\right)$ in the first term of $H_{1}$ describes the intra-channel scattering and is responsible for the localization of that channel, whereas the function $S_{n, m}\left(x_{i}\right)$ in the second term of $H_{1}$ represents the inter-channel hopping and, therefore, facilitates the lateral transport. If we define the average scattering strength as $\left\langle S_{n, m}\left(x_{i}\right) S_{n . m}^{*}\left(x_{i}\right)\right\rangle=\left(W^{2} / 12\right) F_{n, m}$ with $F_{n, m} \equiv \sum_{z}\left|\varphi_{n}^{*}(z) \varphi_{m}(z)\right|^{2}$, it is the relative magnitude of the diagonal and off-diagonal matrix elements of $F_{n, m}$ that reflects the competition between the two scattering processes. The off-diagonal matrix elements, $F_{n, m}$, actually measure the degree of the overlap between the channels $n$ and $m$. Thus, the distribution of $F_{n, m}$ dictates the transport behaviour of the system [4]. The diagonal matrix element, $F_{n, n}$, is nothing but the inverse participation ratio of the channel wavefunctions. The magnitude of $F_{n, n}$ shows the degree of localization of channel $n$. For an extended channel, $F_{n, n}$ is of the order of $1 / N$, whereas for a strongly localized channel, $F_{n, n}$ is close to unity. In fact, for the anisotropic hopping systems, it is easy to see that $F_{n, m}=1 / N$ for all matrix elements. It is important to point out that it is the presence of the off-diagonal long-range order in $F_{n, m}$ that is responsible for the ergodic transport behaviour in the channel space for anisotropic hopping systems. For randomly layered media with isotropic randomness, $F_{n, m}$ is dominated by the diagonal terms, $F_{n, n}$, and there does not exist off-diagonal long-range order in $F_{n, m}$. It is the absence of off-diagonal long-range order that makes the transport nonergodic in randomly layered media with isotropic randomness. 

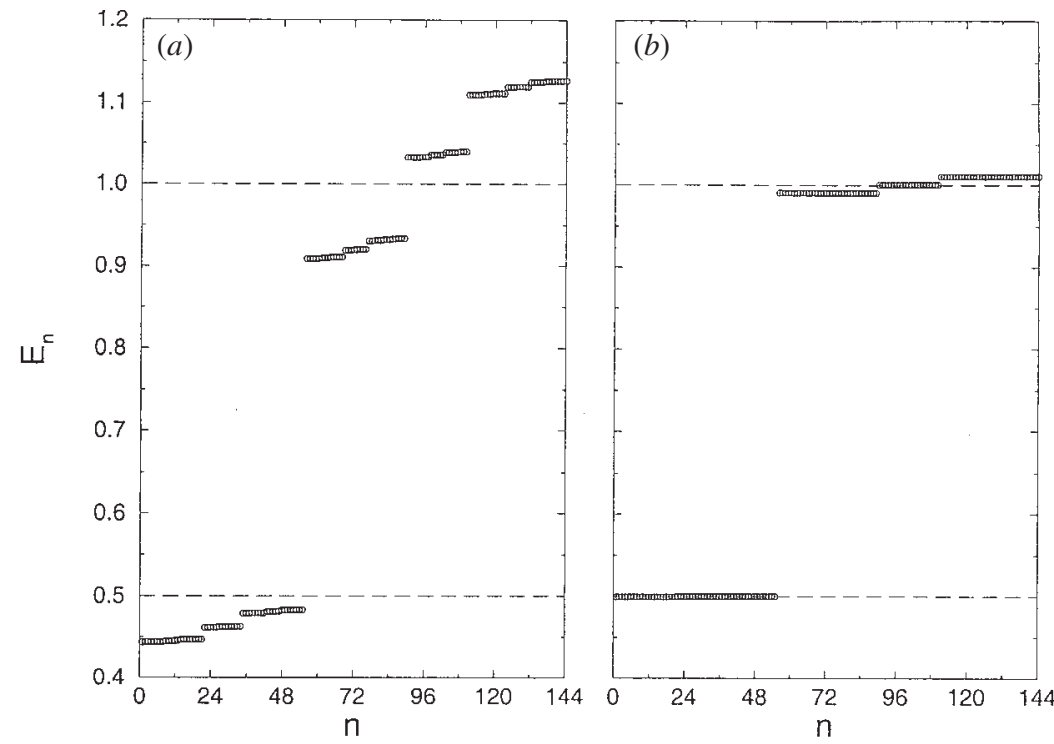

Figure 1. Channel energies in the ninth generation of the Fibonacci sequence with $\varepsilon_{A}=1$, $\varepsilon_{B}=0.5$, and $(a) t_{z}=0.1,(b) t_{z}=0.01$.

\section{Numerical results}

To study the structure of $F_{n, m}$ in quasiperiodically ordered layered media with isotropic randomness we have set $\varepsilon_{A}=1$ and $\varepsilon_{B}=0.5$ as the layer energies. The general features obtained here do not depend on the choice of parameters. In order to obtain the maximum information from our finite-sized calculations, we limit our study to $t_{z} \ll 1$ so that all the channels are propagating in the $x$ direction [4]. It is well known that the spectrum for the channel energies in such systems possess self-similar structures of Cantor type [7]. However, for the convenience of readers as well as our discussions, we plot in figures $1(a)$ and $(b)$, the spectra $\left\{E_{n}\right\}$ in a ninth-generation Fibonacci sequence with $N=144$ layers for $t_{z}=0.1$ and 0.01 , respectively. The corresponding functions of $F_{n, m}$ are also plotted in figures $2(a)$ and $(b)$, respectively. Since the channel wavefunctions, $\varphi_{n}(z)$, are critical and possess large spatial fluctuations, the function $F_{n, m}$ also exhibits self-similar structures similar to those of channel energies. Large fluctuations in $F_{n, m}$ appear both in the diagonal and the off-diagonal parts. Compared with the case of randomly layered media with isotropic randomness and anisotropic hopping systems, the fractal-like behaviour of $F_{n, m}$ is intermediate between the two. By comparing figures $2(a)$ and $(b)$, we also find that when $t_{z}$ becomes smaller the fluctuations in $F_{n, m}$ are larger. This is a result of smaller overlap between channel wavefunctions, which in turn makes the lateral transport less favourable. In order to quantify the fluctuations in $F_{n, m}$ and the degree of channel overlap, we plot in figure 3 the average participation ratio (PR) of $F_{n, m}$ as a function of the layer number, $N$, for three different $t_{z} s: 0.1$ (squares), 0.01 (circles) and 0.001 (diamonds) up to the eleventh generation. To calculate the averaged PR, we first evaluate the PR for a given channel, $n$, by using the expression $(\mathrm{PR})_{n}=\left(\sum_{m=1}^{N} F_{n, m}\right) / \sum_{1}^{N}\left(F_{n, m}\right)^{2}$ and then we average over all $n$, i.e. the averaged $\mathrm{PR}=\sum_{n=1}^{N}(\mathrm{PR})_{n} / N$. It is interesting to point out that, apart from systematic oscillations due to the finite-sized effect, the so-obtained average PR can be well described by a power law, $N^{\alpha}$, with $\alpha=0.649,0.552$ and 0.436 for $t_{z}=0.1$, 0.01 and 0.001 , respectively. Indeed a smaller $t_{z}$ leads to larger fluctuations in $F_{n, m}$ and a 

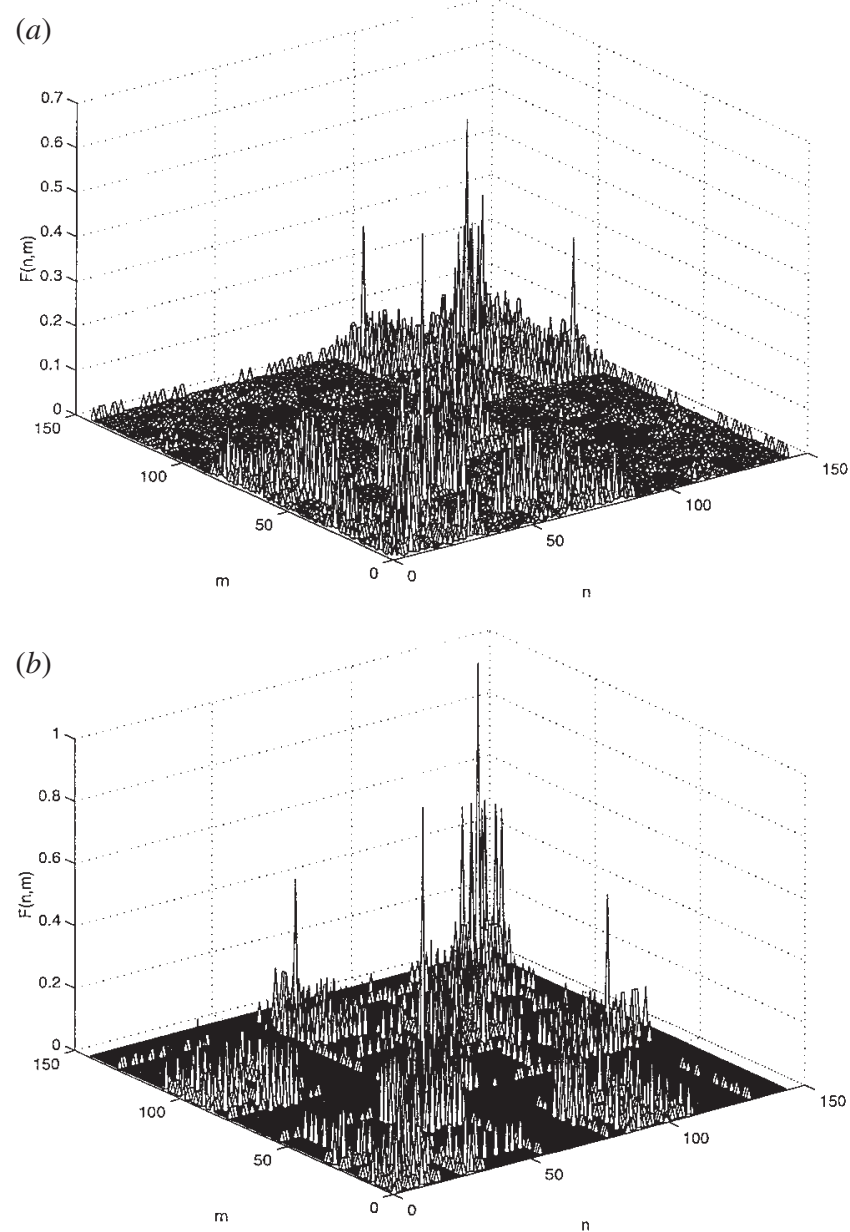

Figure 2. Function $F_{n, m}$ for the same parameters used in figure 1.

smaller averaged PR. Also plotted in figure 3 are the results of randomly layered media with isotropic randomness with layer randomness $W_{1}=1$ and $t_{z}=0.1$ (in triangles) and anisotropic hopping systems (the dashed-dotted curve). A flat line in the randomly layered media with isotropic randomness shows precisely that all channels are strongly localized and there exist no off-diagonal long-range order in $F_{n, m}$. On the other hand, in the case of anisotropic hopping systems, we have the averaged PR equals to $N$ as a result of off-diagonal long-range order, i.e. $F_{n, m}=1 / N$ due to the complete overlap of all channel wavefunctions. In the case of quasiperiodically ordered layered media with isotropic randomness, a power-law behaviour in the averaged PR implies a power-law correlation in the off-diagonal matrix elements of $F_{n, m}$. Since the structure of the matrix $F_{n, m}$ dictates the lateral transport of a layered medium when isotropic randomness is introduced, we expect a different kind of wave transport in quasiperiodically ordered layered media with isotropic randomness.

To investigate the lateral transport, we study the finite-size scaling behaviour of the average number of occupied channels in the asymptotic region by using the recursive Green's function method [11]. In the recursive Green's function calculations, we connect a quasiperiodically 


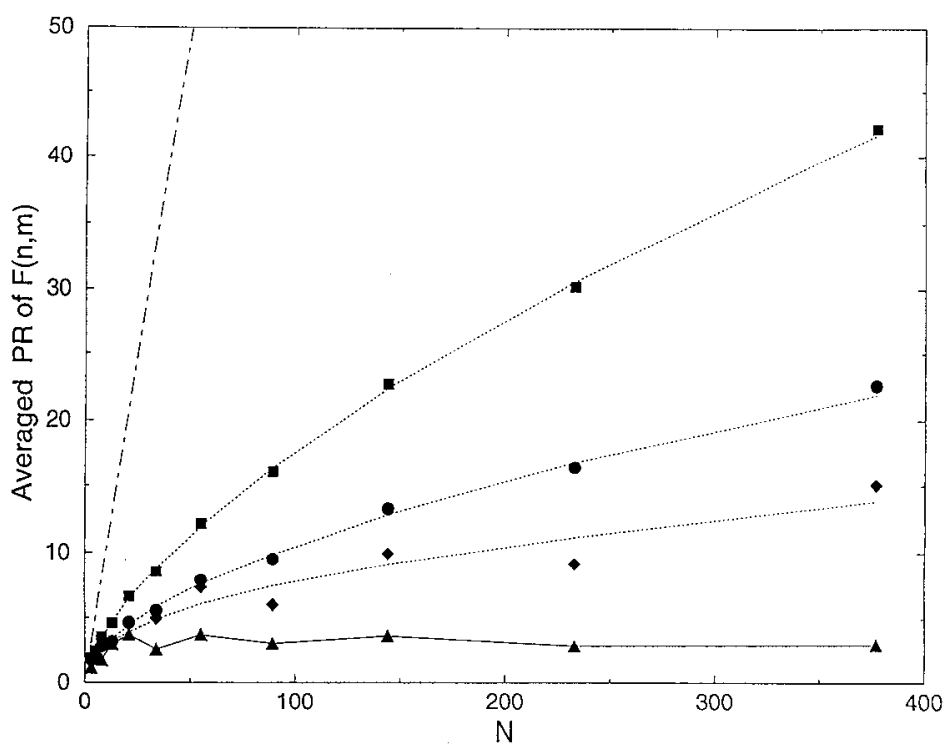

Figure 3. Average PR of $F_{n, m}$ for eleven generations of the Fibonacci sequence with $\varepsilon_{A}=1$, $\varepsilon_{B}=0.5$, and $t_{z}=0.1$ (squares), $t_{z}=0.01$ (circles), $t_{z}=0.001$ (diamonds). The 'triangles' and the dashed-dotted curve are the results for randomly layered media with isotropic randomness and anisotropic hopping systems, respectively.

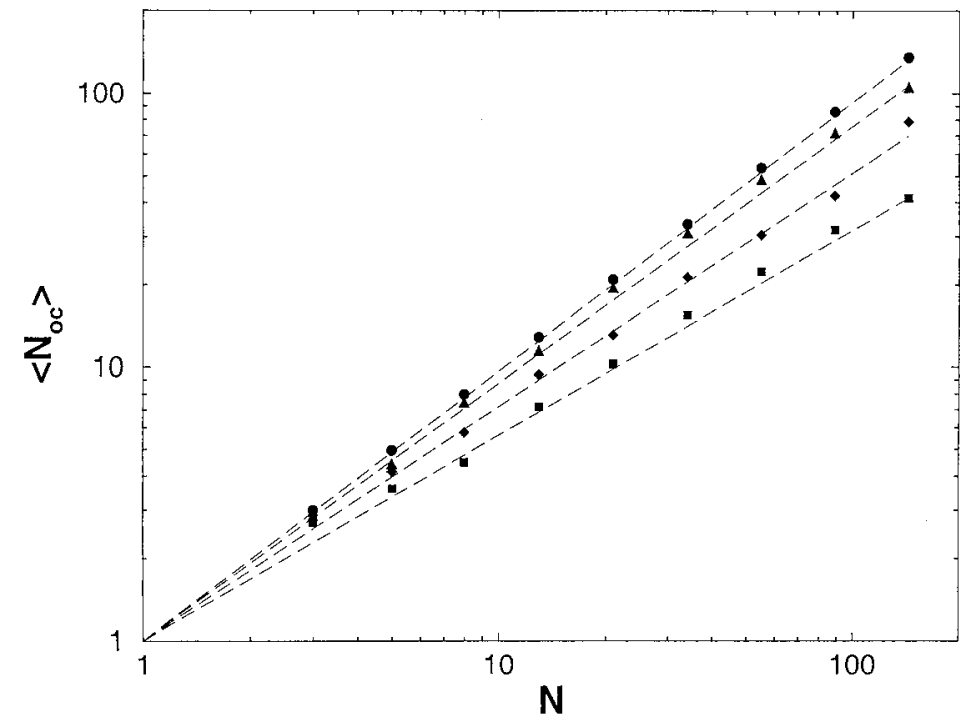

Figure 4. At $E=0$ and $W=1$, the average number of occupied channels is plotted as a function of layer number $N$ up to nine generations of the Fibonacci sequence with $t_{z}=0.1$ (circles), 0.01 (triangles), 0.001 (diamonds) and 0.0001 (squares).

ordered layered medium with isotropic randomness sample with $N$ layers in the $z$ direction and an $L$ length in the $x$ direction to two pure leads on both sides of the sample. These leads have the same layer configuration and anisotropy in $t_{x}$ and $t_{z}$ but $W=0$. The transmission amplitudes, $t_{n m}$, with incoming waves at the $m$ th channel and outgoing waves at the $n$th channel form an 
$N \times N$ matrix and can be calculated numerically with the use of the recursive Green's function method [11]. In our calculations, a periodic boundary condition is used in the $z$ direction. It has been shown analytically that when sample length $L$ is larger than some equilibration length, $L_{e q}, t_{n m}$ reaches an asymptotic region and the ratios $t_{n m}(L) / t_{n \lambda}(L)$ become $L$ independent [3]. From this relation, we can obtain the relative occupation probability in the $n$th outgoing channel as $\theta_{n}(L)=\left|t_{n m}(L)\right|^{2} / \sum_{n=1}^{N}\left|t_{n m}(L)\right|^{2}$, which is independent of the incoming channel $m$ [3]. In the asymptotic region, the statistics of $\theta_{n}(L)$ are stationary and the relative channel occupation distribution can be obtained by taking the configuration average of isotropic randomness with a fixed sample length, $L$, i.e. $\bar{\theta}_{n} \equiv\left\langle\theta_{n}(L)\right\rangle_{L>L_{e q}}$. The same distribution of $\theta_{n}$ can be obtained in a fixed configuration with different $L>L_{e q}$ [4]. The average number of occupied channels is defined as $N_{o c}=1 /\left(\sum_{n} \bar{\theta}_{n}^{2}\right)[2,4]$. It is known that for anisotropic hopping systems, $N_{o c}=N$ and the transport is ergodic and behaves like an isotropic 2D system, whereas for randomly layered media with isotropic randomness, $N_{o c}$ approaches a constant when $N$ is sufficiently large. The transport is nonergodic and the system is 1D-like [4]. In our study of quasiperiodically ordered layered media with isotropic randomness, without losing generality, we have chosen $E=0$ and $W=1$ and calculated $N_{o c}$ up to nine generations of Fibonacci sequence $(N=144)$ with four different values of $t_{z}$. The results for $t_{z}=0.1,0.01,0.001$ and 0.0001 are shown as solid circles, triangles, diamonds and squares, respectively, in figure 4 in a log-log plot. These data points can be well fitted by four straight lines. This implies a fractal-like dependence between $N_{o c}$ and $N$, i.e. $\left\langle N_{o c}\right\rangle \approx N^{v}$. The values of $v$ are 0.98 , $0.94,0.85$ and 0.75 for $t_{z}=0.1,0.01,0.001$ and 0.0001 , respectively. These results confirm our prediction that the lateral wave transport in quasiperiodically ordered layered media with isotropic randomness carries a fractal dimension and its dimensionality decreases with the decreasing of layer coupling.

\section{Discussions}

The existence of a fractal dimension in the lateral transport of quasiperiodically ordered layered media with isotropic randomness is a direct consequence of the fractal-like structures in the scattering function, $F_{n m}$, in the channel space, which in turn is a result of critical-type channel wavefunctions in quasiperiodically ordered layered media. If we use the dimensionless parameter $\gamma \equiv t_{z} /\left|\varepsilon_{A}-\varepsilon_{B}\right|$ to measure the degree of channel overlap, in the limit of infinite $\gamma$, i.e. $\varepsilon_{A}=\varepsilon_{B}$, the value of $v$ approaches unity and the system crosses over to anisotropic hopping systems. When $\gamma$ (or $t_{z}$ ) is reduced, the value of $v$ also decreases as a result of smaller channel overlaps. The system moves towards randomly layered media with isotropic randomness. This is also indicated by the decreasing value of $\alpha$ in figure 3. However, it should be pointed out that the fractal dimension we have found for the average PR of $F_{n, m}$ in figure 3 is different from that of the average number of occupied channels, $\left\langle N_{o c}\right\rangle$, shown in figure 4 , although they both decrease with a decreasing $t_{z}$. The reasons for this are the following. Since the matrix $F_{n, m}$ depends only on the dimensionless layer energies, $\varepsilon_{A} / t_{z}$ and $\varepsilon_{B} / t_{z}$, and the quasiperiodic ordering of the layers, it is an intrinsic property of the system, i.e. it does not depend on the incoming wave energy $E$. However, in the presence of isotropic randomness, the scattering amplitudes of both intra- and inter-channel scatterings do depend on the wave energy $E$. For instance, it has been shown that, in the Born approximation, the transition probability from channel $m$ to channel $n$ has the form $w_{n, m}=\pi W^{2} / 6 v_{m} \rho_{1 D}\left(E-E_{n}\right) F_{n, m}$, where $v_{m}=\mathrm{d} E / \mathrm{d} k_{m}$ is the channel velocity and $\rho_{1 \mathrm{D}}$ is the density of states in one dimension [3]. It has also been shown that the channel with a channel velocity close to 2 has large probability to occur due to long localization length [2,3]. Since the transmission matrix $t_{n m}$ depends on the transition probability between channels as well as the channel localization length [3], it is expected that the fractal dimension 
of $\left\langle N_{o c}\right\rangle$ also depends on the wave energy $E$ and is entirely different from that of $F_{n, m}$. However, it is the fractal behaviour of $F_{n, m}$ that gives rise to a fractal behaviour in $\left\langle N_{o c}\right\rangle$. Except for the above hand-waving arguments, the precise relationship between the fractal dimensions of $F_{n, m}$ and $\left\langle N_{o c}\right\rangle$ is not understood. This remains to be an interesting problem for further study.

Finally, we would also like to point out that in some ordered quasiperiodic systems the fractal dimension of quantum diffusion can also be varied by adjusting the hopping parameters in the system [12]. However, the model we considered here is very different from ordered quasiperiodic systems. In quasiperiodically ordered layered media with isotropic randomness, the fractal-type ergodicity we have studied here is in channel space. It originates from the interplay between quasiperiodic ordering in the layering direction and the presence of isotropic randomness in the system. This unique feature of quasiperiodically ordered layered media with isotropic randomness does not exist in any ordered quasiperiodic systems.

\section{Conclusions}

In conclusion, we have studied the lateral transport of waves in quasiperiodically ordered layered media with isotropic randomness. In the case of the Fibonacci sequence, the finitesize scaling results of the channel occupation number indicate that such systems carry a fractal dimension for the lateral wave transport. Furthermore, the dimensionality can be tuned continuously by varying the strength of the layer coupling. The transport behaviour of such systems falls between the transport in two other known anisotropic systems, i.e. randomly layered media with isotropic randomness and ansitropic hopping systems.

\section{Acknowledgment}

The authors thank the support of Hong Kong Research Grant Council grant no HKUST 686/96P.

\section{References}

[1] Xue W, Sheng P, Chu Q J and Zhang Z Q 1989 Phys. Rev. Lett. 632837

Zhang Z Q, Chu Q J, Xue W and Sheng P 1990 Phys. Rev. B 424613

Chu Q J and Zhang Z Q 1993 Phys. Rev. B 4810761

[2] Sheng P and Zhang Z Q 1995 Phys. Rev. Lett. 741343

[3] Zhang Z Q and O K C 1996 Phys. Rev. B 53 R11 917

[4] Chan M C, Zhang Z Q and Woo P W 1998 Phys. Rev. B 57 R8071

[5] Ramos C A, Caceres M O and Lederman D 1996 Phys. Rev. B 537890

[6] Apel W and Rice T M 1983 J. Phys. C: Solid State Phys. 16 L1151 Li Q, Soukoulis C M, Economou E N and Grest G S 1989 Phys. Rev. B 402825

Zambetaki I, Li Q, Economou E N and Soukoulis C M 1996 Phys. Rev. Lett. 763614

Li Q, Katsoprinakis S, Economou E N and Soukoulis C M 1997 Phys. Rev. B 56 R4297

[7] Kohmoto M and Banavar J R 1986 Phys. Rev. B 34563

Kohmoto M, Sutherland B and Tang C 1987 Phys. Rev. B 351020

Kohmoto M, Sutherland B and Iguchi K 1987 Phys. Rev. Lett. 582436

[8] Fu X, Liu Y, Zhou P and Sritrakool W 1997 Phys. Rev. B 552882

[9] Macia E and Dominguez-Adame F 1996 Phys. Rev. Lett. 762957

[10] Nazareno H N, de Brito P E and da Silva C A A 1995 Phys. Rev. B 51864

[11] Lee P A and Fisher D 1981 Phys. Rev. Lett. 47882

[12] Yuan H Q et al 1999 Preprint cond-mat/9912176 